\title{
General Dentistry
}

National Cancer Institute

\section{Source}

National Cancer Institute. General Dentistry. NCI Thesaurus. Code C63745.

A dentist involved in the treatment of the oral facial disease who has not limited his/her practice to any dental specialty. 